\title{
LA ARQUITECTURA DE \\ LAS ORGANIZACIONES EN EL SIGLO XXI
}

Peter José Schweizer ${ }^{1}$

\section{RESUMEN}

Este artículo se propone analizar la arquitectura de las organizaciones necesaria para el siglo XXI y orientado a las personas, al ambiente, ajustada a las decisiones políticas y adecuadas a la administración. Propone la humanización en el proceso productivo y destaca la importancia de las universidades en ese proceso de cambio.

Palabras clave: organizaciones, medio ambiente, planificación, administración
ABSTRACT:

This article intends to discuss the architecture of the organizations for the 21 st century and is oriented to people, the environment; it is also adjusted to proper administration and political decisions. It proposes the humanization of the production process and highlights the importance of universities in this process of change.

Key words: organization, environment, planning, administration

\section{INTRODUCCIÓN}

La aplicación del término arquitectura en el campo de estudios de las organizaciones merece una explicación previa. En su origen, arquitectura significa una concepción de un espacio que se realiza a través de una determinada construcción estética y funcional, con áreas delimitadas y destinadas a cubrir funciones sociales y/o necesidades humanas. Las organizaciones a que se hace aquí referencia pueden ser las productivas $u$ otras como es el caso de las uni-

\footnotetext{
'Pontificia Universidad Católica del Ecuador. Facultad de Arquitectura. Quito, Ecuador (pschweizer604@yahoo.com.br).
} 
versidades y sus facultades para la formación de dirigentes, líderes, profesionales en distintos campos.

La arquitectura, como una de las más antiguas manifestaciones de la cultura humana, nos ha enseñado, a lo largo de los siglos, la importancia de que espacios construidos deben ser creados y diseñados en función de las necesidades y de las aspiraciones humanas. Asimismo, las organizaciones deben dejar de buscar adaptar las personas a sus objetivos y pasar a atender las necesidades humanas y, siempre que sea posible, sus aspiraciones.

Bajo el título de"arquitectura organizacional" han sido presentadas contribuciones destacadas que, por ejemplo, la definieron como"el arte de modelar el espacio organizacional para satisfacer necesidades y aspiraciones humanas"2. Esta definición aún es incompleta dado que la arquitectura de las organizaciones puede comprender, además de la propia "arte de modelar", algo que antecede a esa arte que es el propio principio de la razón que define el sentido y la finalidad de una organización. La arquitectura organizacional, además de ser un arte, comprende, también la existencia de una ciencia que permite hacer del "espacio organizacional" una expresión de la historia del conocimiento y de las expe- riencias humanas. Finalmente, la arquitectura organizacional también demanda un conocimiento instrumental que presupone la existencia de la técnica. A través de esa técnica son establecidas las bases para la construcción operacional de una organización específica y son realizadas las modificaciones que esta demanda a lo largo del tiempo.

En el presente estudio se pretende ampliar la definición de arquitectura de las organizaciones hacia la "filosofía, el arte, la ciencia y la técnica de crear y operar espacios organizacionales para atender las necesidades y aspiraciones sociales y humanas". Esta visión de las organizaciones, más integral u "holística", permite señalar algunos enfoques nuevos para el siglo XXI. Vivimos una nueva era que demanda de modificaciones cualitativas en el funcionamiento de la sociedad humana, en armonía con los requerimientos ambientales y las propuestas para el desarrollo sustentable. Por supuesto, demanda cambios sustantivos en la formación de los profesionales en las universidades.

Actualmente la sociedad se encuentra frente a una importante encrucijada de la historia en la cual la humanidad necesita urgentemente redefinir cómo pretende convivir con la naturaleza, con las necesidades de pro-

${ }^{2}$ Nadler, David A. y otros (1994). Arquitetura organizacional: chave para a mudanca empresarial. Río de Janeiro: Editora Campus. 
ducción y consumo y consigo misma en el futuro inmediato. La devastación a que fueron sometidos los territorios, acompañada de la contaminación sin precedentes de los ríos y océanos, así como del aire, y el aumento de la pobreza y de la miseria, demandan reflexiones sobre el nuevo rol que deberán cumplir las organizaciones, entre ellas las universidades, para cambiar, de forma considerable, este panorama en los próximos años.

Otro tipo de devastación está vinculado al ámbito de la convivencia humana. Convivencia de las personas en las escalas familiar, comunitaria, urbana, regional, nacional e internacional, que después de dos guerras mundiales, evolucionó marginalmente hacia una situación mejor para las personas, dados los desequilibrios ambiental y de distribución de los ingresos. Es lo esperado después de tantos ejercicios de irracionalidad, sea en búsqueda desmesurada de la ganancia en las ventajas individuales, realizados por la humanidad en el pasado siglo y principios del presente.

Al mismo tiempo en que ocurría la devastación aquí referida, las organizaciones eran creadas y han sido sistemáticamente destruidas en nombre del mercado, de la producción masiva y de la búsqueda de un consumo sin límites. Esto solo ha logrado agravar las relaciones de los seres humanos con la natura- leza y con sus propias sociedades. En el campo de las organizaciones el problema más evidente que se puede observar, sin que se busque comprender sus causas reales, es el desempleo crónico que lleva a millones de personas a la marginalidad y a muchos países al caos y preocupantemente más grave en los extractos de la población joven.

Aparentemente eliminado el maniqueísmo político que venía operando las relaciones internacionales durante casi medio siglo, fue ganando cuerpo una concepción denominada neoliberal que exacerbó aún más la propuesta de un mercado sin fronteras y de la producción masiva con agresión creciente a la naturaleza. La nueva propuesta, imposición dominante de un mundo manejado exclusivamente por un concepto de mercado sin fronteras, solo logra abreviar aún más el tiempo de existencia de los recursos naturales no renovables y la reducción de aquellos que podrían ser renovables y que se pierden ante el desperdicio y la irracionalidad en su uso.

La búsqueda de una función que sea esencial y al mismo tiempo sustentable para las organizaciones, en el siglo $X X I$, es el objetivo de estas reflexiones que estarán orientadas hacia una propuesta de una nueva arquitectura de las organizaciones que contemple un proceso de producción restringido a un nivel cualitativo de suficiencia y un mer- 
cado delimitado por su propia esencia. ${ }^{3}$ Lo fundamental que debe ser proyectado consiste en el hecho de una nueva arquitectura de las organizaciones capaz de concebir organizaciones para que sean subsidiarias y complementarias a las necesidades humanas y no al contrario, como está ocurriendo hasta los días presentes.

Merece enfatizarse que cuando hablamos hoy de "organizaciones" normalmente esto se refiere a un tipo de entidad social dirigida hacia la consecución de finalidades productivas. Significa una unidad de transformación de materia prima o de informaciones en productos acabados o servicios, en la forma de un bien concreto o también de información. En este documento el término"organización" gana una acepción más amplia, de acuerdo con lo propuesto por el sociólogo brasileño Alberto Guerreiro Ramos en su libro "La nueva ciencia de las organizaciones". ${ }^{4}$

En efecto, en un encuentro promovido por la Fundación Getúlio Vargas (Río de Janeiro), en septiembre de 1982, a propósito de la vida y obra del profesor Guerreiro Ramos, uno de los participantes aclaró de forma explícita cual es el concepto sustantivo de organización: "la organización a la que él (Guerreiro Ramos) se refiere no es solo la organización formal o economicista. Es mucho más que eso, es el orden de la vida humana asociada, es la organización de las interacciones humanas. ${ }^{5}$ De esa forma, y para los propósitos de este artículo, el concepto de organización trasciende a los límites productivos y a las relaciones meramente funcionales.

\section{ARQUITECTURA DE LAS ORGANIZACIONES Y LAS PERSONAS}

El trabajo generado en cualquier organización, por ejemplo en una universidad, es realizado por personas y, por lo menos por definición, debería estar dirigido a ellas. Esta es la razón por la cual la relación entre la concepción, el proyecto (estructuras, sistemas y procesos), así como el funcionamiento de las organizaciones, y las personas que las operarán, merecen ser evaluados, y muy probablemente, rediseñados. Las universidades de nuestros días, aprisionada por sus conceptos funcionales y procesos internos, termina por capacitar profesionales para los paradigmas del pasado y en algunos casos se cierran

\footnotetext{
${ }^{3}$ Ramos, Alberto Guerreiro (1981). A nova ciência das organizações: uma reconceituação das riquezas das nações. Río de Janeiro. Editora Fundação Getúlio Vargas.

${ }^{4}$ Ramos, Alberto Guerreiro (1981). Op. Cit.

${ }^{5}$ Resende, Ubiratan Simões. Cuarto Panel - "A teoria da delimitação dos sistemas sociais". Revista de Administração Pública-RAP. Río de Janeiro, FGV, abril - junio de 1983.
} 
para los cambios demandados por nuevos requerimientos para el bienestar de la sociedad.

La universidad necesita transformar su estructura piramidal de poder en una estructura horizontal, interdisciplinar, en que el saber pueda transitar de un campo para el otro sin limitaciones, para formar un profesional con una visión holística. Un profesional que tenga la capacidad de operar y participar activamente de la transformación del mundo como lo indica seguidamente el Papa Francisco al proponer la "construcción de puentes" incluso para el conocimiento y saber humano.

En la actualidad, la concepción estructural y operacional de la arquitectura de las organizaciones tiene como finalidad adecuar las personas al trabajo que estas les exigen. Las personas, conjunto de individuos, de acuerdo con esta concepción, corresponden solamente a un componente del mecanismo organizacional junto con las máquinas, los equipos, los sistemas y las instalaciones requeridas por la operación. Por constituirse en la parte más compleja y de difícil estandarización, a pesar de los esfuerzos que para este fin se realizan a través del entrenamiento de contenido conductual, el individuo viene perdiendo sistemáticamente su posición en pro de las máquinas. El profesor, por ejemplo, pasa a ser un operador de computadora y poco más. La sustitución de personas, en las organizaciones, por mecanismos y sistemas, ha permitido al capital una autarquización para liberarse de legislaciones e instituciones como los sindicatos, que se proponen defender las personas y los trabajadores de las organizaciones que los utilizan.

Además, esta situación ha minado progresivamente uno de los fundamentos del actual sistema productivo: las relaciones capital - trabajo. Sin opciones, o mejor dicho, con cada vez menos opciones de empleo, la fuerza motriz de las economías, las personas, tienen solo dos salidas: convertirse en generador de capital o alienarse del mercado de trabajo, o sumándose a una creciente cantidad de personas "desajustadas" a los requerimientos del mercado de trabajo. La primera alternativa se expresa en los movimientos por la creación de micro y pequeñas empresas. A su vez, la segunda, que en realidad representa la propia "falta de alternativa", viene alienando personas del mercado, ya sea en el medio rural, en los minifundios de subsistencia, y también en las ciudades, principalmente en las grandes, donde el aumento del número de personas en situación de pobreza o extrema pobreza genera hoy una marginalidad consolidada y orgánica, poco reactiva o mismo inerte a las principales políticas de desarrollo económico y social. 
La mayoría de los que actualmente diseñan las organizaciones las proyectan en términos de un organigrama formal y un concepto de proceso productivo en el cual las unidades de trabajo están asociadas, así como se definen las tareas que deben cumplir. Una universidad, de esa forma, tiene que ofrecer conocimientos (normalmente científicos, artísticos y técnicos) y diplomas que correspondan a ellos. No obstante, esto no permite identificar las personas que componen tales unidades y carreras en términos de sus personalidades, sus relaciones propias y relaciones con el poder, la iniciativa individual, el compromiso de cada uno con los objetivos de la organización, además de otros factores que expresan el grado de complejidad del desempeño humano en un mundo globalizado.

Los modelos clásicos de concepción de las organizaciones, cerrados e inflexibles, mecanicistas u orgánicos, están siendo sustituidos por una visión de la organización como un sistema de interacción entre personas. Sin embargo, la idea de un ser humano limitado solamente a un factor o componente de la producción, no permitió, hasta hoy, una evolución práctica en el rumbo de una nueva arquitectura capaz de "diseñar" y concebir sistemas organizacionales en bases sociales.

La máquina se convirtió y es cada vez más importante que aquellas personas que las organizaciones aún están obligadas a mantener se subordinan incondicionalmente temerosos de la siempre presente y creciente amenaza del desempleo. Es exactamente el desempleo que hoy afecta desde el trabajador hasta el más alto ejecutivo con sofisticada formación profesional y académica, además de afectar el funcionamiento político de las entidades que los representan, que ya no luchan por mejores condiciones para el trabajador, sino apenas por mantener el empleo en las condiciones impuestas por el empleador.

En realidad, la modernidad y el avance tecnológico representaron un retroceso importante en las condiciones que se le impusieron al trabajador que pasó a ser considerado como parte de una clase privilegiada solamente por disponer y poder mantener algún tipo de empleo el mismo que sea con una remuneración decreciente.

\section{LA ARQUITECTURA DE LAS ORGANIZACIONES Y EL DESAFÍO AMBIENTAL}

El progresivo agotamiento de los recursos naturales no renovables y la ex- tinción de parte de los recursos renovables consumidos por los excesos huma- 
nos, como es el caso de muchas especies de árboles y animales que ya no existen por la explotación extensiva realizada en siglos pasados, constituye un testimonio vivo de la forma irracional como vienen operando las organizaciones.

En este contexto se observa la existencia de una cantidad significativa de organizaciones productivas, como es el caso de las industrias contaminantes y fraccionadoras del ambiente natural y cultural. Esto lleva a afirmar que la ecuación del mercado: producir mucho y de mala calidad, con obsolescencia acelerada o programada y al costo ambiental que fuera necesario para maximizar la ganancia, debe ser frenada y revista en sus fundamentos. Hoy ya se observan movimientos en esa dirección. Las universidades, en ese contexto, tienen gran importancia en la creación de una conciencia colectiva tanto local como planetaria.

Los excesos de confianza en el proceso técnico asociado al laissez-faire hace que las clases dirigentes mundiales consideren que el problema del agotamiento de los recursos naturales no renovables pueda ser satisfactoriamente superado a través de la sustitución de nuevas fuentes de energía, nuevas tecnologías y modernos medios que se adecuarán "naturalmente" al conjunto de las necesidades humanas erróneamente caracterizadas como "demandas del mercado".

Ya en los años 1970 ya nos alertaba el profesor y sociólogo Alberto Guerreiro Ramos sobre el hecho que"en los centros de investigación de vanguardia predomina el consenso que la modernización y el desarrollo son inseparables de las prácticas predatorias, nocivas a la vida humana y a las funciones autorregulables del ambiente natural". ${ }^{6}$

Sin embargo, en el último final de siglo ya no existía indicador que la humanidad, a través de las clases dirigentes, esté imponiendo límites a los procesos de "modernización"y de lo que se entiende por desarrollo. Este sigue siendo la meta final a alcanzar para la superación de los males crónicos que nos afectan de forma creciente, entre los cuales se destacan el aumento del desempleo y de la violencia contra el ambiente y, más que todo, en contra del ser humano.

El desarrollo que los países de América Latina han perseguido en los últimos años es resultante de las concepciones tradicionales, concepciones todavía mecanicistas sobre el funcionamiento del sistema económico. Las deformaciones que fueron y se están acumulando a lo largo del camino,

\footnotetext{
${ }^{6}$ Guerreiro Ramos, Alberto. "Límites de la Modernización". Artículo publicado en: Jornal do Brasil. Río de Janeiro, mayo 20 de 1979.
} 
hacen con que muchos filósofos modernos pierdan las esperanzas en la racionalidad humana como medio capaz de revertir el funcionamiento del proceso productivo y, consecuentemente, de la concepción y el funcionamiento de las organizaciones. Por lo tanto, es urgente revisar la arquitectura de las organizaciones presentes para poder evaluar efectivamente las repercusiones del funcionamiento destructivo y desintegrador sobre el ambiente natural y cultural tanto en el campo cuanto en la ciudad.

\section{ARQUITECTURA DE LAS ORGANIZACIONES Y LA DECISIÓN POLÍTICA}

Cualquier intento de cambio del panorama existente hoy en América Latina requiere voluntad, movilización social y decisión política. Aquí se entiende por política el arte y la ciencia de dirigir las relaciones sociales y tomar decisiones en una organización privada o gubernamental.

En la actualidad los países en desarrollo, como los de América Latina, están buscando insertarse de forma inconveniente en el ambiente de la denominada globalización que ha sido difundida como un nuevo paradigma para alcanzar el progreso económico y social. El mundo es ahora visto como un mercado global y los países pierden sistemáticamente su identidad en función del capital sin fronteras y sin ningún tipo de compromiso nacional, regional y mucho menos social.

Además, el ciudadano, el individuo, continúa siendo el mismo, vinculado a la familia, a su comunidad local y a todos los valores que siguen orientando sus relaciones sociales. El hecho de tener acceso a canales de televisión de otros países o a la red de Internet, así como el acceso a productos importados de otros países, transforma al ser humano en un consumidor mundial automáticamente incorporado a través de la globalización. Esa globalización aún no significa, al nivel de vida y cotidianidad de las personas, alteraciones sustanciales en lo relacionado con el aumento de cultura y de ingresos y menos aún de mejoras en las condiciones de vida material o espiritual. Un panorama de inseguridad física y económica está generando una creciente desconfianza en las organizaciones, especialmente en aquellas de carácter político que deberían representar una concentración de fuerzas para mejorar la vida individual y colectiva.

Una nueva arquitectura de las organizaciones debe ser el fruto de una propuesta, ambientalmente factible y de 
políticas concebidas en función de las personas y no de los componentes económicos y comerciales que hoy predominan. Esto para que no se pierda el tenue convencimiento en las cualidades de la democracia, tan discutidas en nuestros países en función de resultados todavía poco convincentes. La economía debe subordinarse a los requerimientos políticos y sociales de toda la sociedad y no en la dirección inversa, como predomina en los días actuales.

\section{ARQUITECTURA DE LAS ORGANIZACIONES Y LA ADMINISTRACIÓN DEL SIGLO XXI}

Una nueva arquitectura para las organizaciones que conforme un marco de referencia para la acción humana en este siglo XXI debe considerar algunos principios generales que sometemos a consideración de los que tienen interés en el tema.

\section{Una estrategia centrada en las perso- nas}

\section{- Proponer la planeación con las per- sonas y no para las personas}

Hasta hoy se ha planificado para la construcción de obras físicas, para la construcción de las ciudades, para las regiones, para los países, para las empresas, para atender el mercado. Sin embargo, olvídase de planificar con las personas afectadas. Discursos vacíos sobre una planificación participativa no han logrado obtener resultados concretos para el beneficio de la población en nuestros países. Solamente han escamoteado y legitimado decisiones "de arriba hacia abajo" justificadas bajo el pretexto de una eficiencia técnica, sin demostrar una real eficacia humana y social.

También en las universidades se hace necesario planificar no solamente con los profesores o solo con los dirigentes, pero también con los alumnos y con la participación de representantes de la población local (lo que significa una efectiva práctica de participación comunitaria).

La racionalidad ha sido limitada a la del "mercado", desconociéndose el valor humano, las necesidades de sustentabilidad de la humanidad y de su ambiente natural. El mejor ejemplo de eso ha sido el desempleo al cual han sido sometidas millones de personas en todo el mundo, además de otros millones de personas que jamás tuvieron acceso a un empleo formal, ni urbano ni mucho menos rural.

La planificación con las personas significa, en las organizaciones de este siglo, oír a los interesados y afectados en 
las acciones del gobierno o de las empresas, en la discusión de los asuntos relevantes y en la toma de decisiones que puedan afectar sus vidas. Existen precedentes importantes que nos permiten garantizar la factibilidad de este procedimiento como una posible práctica operacional.

\section{- El proyecto como referencia funda- mental para las iniciativas humanas}

La existencia previa de proyectos centrados en las personas y sustentables ambientalmente debe ser un requerimiento básico para las organizaciones del futuro. Hasta el presente el proyecto ha tenido como foco la rentabilidad de las iniciativas apoyada en las relaciones de costo-beneficio económico; la construcción de elementos físicos; el retorno para los financiadores. Sin embargo el proyecto no se transformó en un recurso organizacional para garantizar a la sociedad la concreción de iniciativas capaces de lograr que la vida humana pueda ajustarse al medio natural que la rodea y le da sustancia. Refiérase no solamente a los proyectos de arquitectura e ingeniería como también a los proyectos económicos y sociales.

El desconocimiento de las organizaciones formales en relación con miles de personas condenadas a una vida de pobreza extrema en asentamientos humanos precarios, en las ciudades (en tu- gurios, invasiones y otros) y en las áreas rurales, demuestra la inexistencia de proyectos que consideren a la población como un todo y no solamente a los grupos privilegiados como se ha hecho hasta ahora. Las universidades tienen también en este caso un papel destacado en la formación de un saber demandado por ese campo orientado al bienestar social.

\section{- La optimización en el uso de la ener- gía y la eliminación de los desechos}

Uno de los aspectos característicos de la sociedad centrada en el mercado ha sido el mal uso de la energía y la creciente generación de desechos. Esto último se convirtió en un requerimiento puesto que del desperdicio pasó a depender la producción de nuevos bienes para la población mundial que tiene acceso a ellos. Entre más rápido un objeto es eliminado y se hace obsoleto, más posibilidades existen para que el denominado mercado se amplíe con nuevas demandas que generen más lucro para las organizaciones privadas que lo producen.

En el caso de la energía ya existe un embrión de conciencia en algunas organizaciones, en distintas partes del mundo, que el manejo de los recursos energéticos en este siglo no debe estar subordinado a la ganancia de las empresas sino a la atención global de la pobla- 
ción, sin que sean afectadas las condiciones ambientales actuales y futuras, condiciones de las cuales dependerán todas las nuevas generaciones.

- Hacer de la satisfacción personal de sus necesidades esenciales (físicas, sicológicas y espirituales) el foco del proceso productivo

La reducción del ser humano a la simple condición de "consumidor"en un mundo considerado apenas como un "mercado globalizado" hizo que la dimensión humana se diluyera. De esta manera, quedaron visibles solo las necesidades biológicas capaces de ser manipuladas psicológicamente a través de la propaganda en donde el comportamiento humano no difiere del animal. Las Universidades son parte de las organizaciones que puede cambiar esta visión deformada que nos ha sido impuesta.

Una nueva arquitectura de las organizaciones centrada en la persona, una Universidad centrada en el alumno, no como consumidor sino como ser integrado de forma holística a la naturaleza, hará que se transformen las relaciones locales, nacionales y mundiales. La satisfacción del ser humano estará armonizada con los requerimientos ambientales globales existentes.

\section{- Construir escenarios de futuro sus- tentables}

Es cada vez más importante planificar mirando hacia el futuro y analizando sus requerimientos básicos o esenciales. Para ello, es necesario la elaboración de escenarios que permitan analizar la posible evolución de situaciones que afectarán a las personas y la estructura ambiental con la cual conviven y de la cual dependen. Las universidades tienen que mirar hacia el futuro y para qué tipo de profesionales la sociedad podrá requerir y no más quedarse estática formando profesionales que no tendrán, al graduarse, ninguna inserción en la sociedad, sea como emprendedores o como empleados, públicos o privados.

\section{Fortalecer la condición del ser hu- mano y del conocimiento humano}

\section{- Sustituir el entrenamiento por la ca- pacitación}

En pasado siglo los seres humanos fueron "entrenados", es decir, mecánicamente condicionados y ajustados para cumplir rutinas predeterminadas donde la inteligencia ha sido sustituida por el ajuste a las tareas (enfoque comportamental) demandadas por las máquinas.

El ser humano no debe recibir un entrenamiento conductual de la misma 
forma como se adiestran animales. Debe ser capacitado para realizar trabajos de interés personal y social. Debe ser despertado hacia un sentido de responsabilidad y solidaridad con sus semejantes, con su comunidad y con la naturaleza, de manera que se armonice con ella en una convivencia sustentable. Las universidades forman personas con un conocimiento universal capaces de aprender y no solo formar técnicos operacionales, hasta porque estos últimos ya no son los mismos, considerándose la multiplicidad de opciones tecnológicas hoy a disposición de las organizaciones permanentemente sumergidas en procesos de cambio.

\section{- Desarrollar nuevos talentos}

Los seres humanos tienen una capacidad increíble de adaptación, de percepción y creatividad que merece ser estimulada no solo para el desarrollo personal, sino principalmente para el desarrollo social y global, en distintos ámbitos organizacionales.

Se avecina una nueva era en la cual no bastan los conocimientos especializados. Es imprescindible promover conocimientos en sintonía con la naturaleza, conocimientos que sean capaces de despertar talentos que tengan condiciones de desarrollar nuevos conocimientos filosóficos, científicos y artísticos y que contribuyan sustantivamente al progreso de la humanidad. Las universidades tienen, sin duda, una responsabilidad primordial en ese aspecto.

\section{- Priorizar una educación holística}

Este es, indudablemente, el campo en el cual es notoria la regresión humana. La educación formal estuvo más orientada al cumplimiento de determinadas rutinas que a la preparación de las personar para la vida, para la convivencia social democrática, para el uso de la creatividad y para lograr la efectiva inserción productiva.

Miseria y segregación, guerras y destrucción ambiental han llegado a niveles nunca antes observados en el mundo, haciendo del ser humano solamente un espectador de las noticias sobre conflictos y sobre ese proceso de desagregación del ambiente físico y del ambiente cultural. Sin una reversión en el ámbito educativo, campo que merece ser repensado completamente, no se logrará alcanzar, una arquitectura organizacional humanizada planteada en este texto.

\section{- Remplazar la competencia por la co- operación}

En el final de siglo XX, cuando la competencia fue llevada a los extremos, fue posible verificar que la disputa en el violento proceso de competitividad pro- 
fesional generó resultados desastrosos en términos humanos y sociales.

Es pues importante determinar una forma de promover la cooperación en la cual los valores humanos sean estimulados, permitan sumar, fortalecerse y no tengan que anularse en una disputa febril por el éxito y por la riqueza material ni mucho menos por destacadas posiciones profesionales.

Una sociedad más justa podrá formarse ahora, con más conciencia política, ecológica y social, con base en la práctica efectiva de la cooperación a nivel individual y social así como en el ámbito de las naciones.

\section{- Reducir controles y fortalecer las responsabilidades}

La herencia de experiencias totalitarias del pasado siglo generó una visión y concepción de que lo fundamental está en la existencia de mecanismos de control para que las personas funcionen como máquinas que garanticen la producción. Esto solo significa que sean eficientes y permitan las ganancias en las empresas. La contribución humana quedó reducida al trabajo asalariado que hoy se encuentra en crisis en todo el mundo en la medida en que las máquinas sustituyen progresivamente el trabajo que realizaban las personas.

Para fortalecer la condición de ser humano será indispensable que este asuma crecientes responsabilidades, individuales y colectivas, para que no sea solamente dependiente de organizaciones públicas y privadas como empleado, sino que sea actor activo en el proceso de construcción de una sociedad mundial solidaria. Se requiere, pues, que cada persona asuma la posición de líder en ese proceso en el cual son actores y no, como en el pasado, espectadores.

Que las universidades amplíen los conocimientos y los espacios para que eso pueda ocurrir. Los desafíos e incertidumbres son muchas pero una cosa es segura: el rol de una universidad, con una arquitectura modificada y ajustada a los requerimientos de esta nueva Era, es sin duda una pieza fundamental en la medida que apunte para los caminos que conducen al futuro, con individuos, organizaciones y sociedades sustancialmente diferentes de lo que se dispone en los días presentes. 


\section{BIBLIOGRAFÍA}

Nadler, David A. y otros (1994). Arquitetura organizacional: chave para a mudanca empresarial. Rio de Janeiro: Editora Campus.

Guerreiro Ramos, Alberto (1981).'A nova ciência das organizações: uma reconceituação das riquezas das nações. Rio de Janeiro. Editora Fundação Getúlio Vargas.

Resende, Ubiratan Simões. Cuarto Panel - "A teoria da delimitação dos sistemas sociais". Revista de Administração Pública - RAP. Rio de Janeiro, FGV, 1983.

Guerreiro Ramos, Alberto. "Límites de la Modernización". Artículo publicado en Jornal do Brasil. Rio de Janeiro, 1979.
Pesca de Acosta, Claudia Adriana y Tovar, Lioval Moreno (2003) - "Una Arquitectura Organizacional Inteligente". Publicado en la Revista Universitaria de Investigación. Caracas.

Guerreiro Ramos, Alberto (1972). "Models of Man and Administrative Theory". Publicado en American Society for Public Administration. Washington. USA.

Guerreiro Ramos, Alberto (1983). "A Nova Ignorância e o Futuro da Administração Publica na América Latina" publicado em la Revista de Administração Publica. Rio de Janeiro. 